\title{
Manejo nutricional actual de la PKU en niños
}

\section{Current nutritional management of PKU in children}

Pág. 205,211

Recibido: $20-05-2020$

Aceptado: 13-07-2020

Stephanie Mendoza Ríos ${ }^{1}$

1. Nutricionista.Trabajador Independiente, Costa Rica.

\section{RESUMEN}

La fenilcetonuria es una enfermedad metabólica hereditaria que requiere de un tratamiento nutricional de por vida el cual pretende mantener los niveles de PHE en sangre bajos para evitar daño neurológico, pero además debe proveer un adecuado aporte de proteína y energía, así como mantener un adecuado estado nutricional desde el momento del diagnóstico (LópezMejía, Vergara-Vázquez \& Guillén-López, 2019). Debido a esto, se pretendió evidenciar el tratamiento nutricional brindado a pacientes pediátricos con $\mathrm{PKU}$ según datos recientes sobre el tema con la presente revisión bibliográfica. Palabras clave: fenilcetonuria, pediatría, omega 3 , microbiota, nutrición, glicomacropéptido.

\section{ABSTRACT}

Phenylketonuria is an inherited metabolic disease that requires nutritional treatment for life, which aims to keep blood levels of PHE low to avoid neurological damage but must also provide an adequate supply of protein and energy, as well as maintain an adequate nutritional state from the moment of diagnosis (López-Mejía, VergaraVázquez \& Guillén-López, 2019). Due to this, the aim of this bibliographic review is to show the nutritional treatment offered to pediatric patients with PKU according to recent data on the subject.

\section{KEYWORDS}

phenylketonuria, pediatrics, omega 3 , microbiota, nutrition, glycomacropeptide.

\section{INTRODUCCIÓN}

Existe un grupo de condiciones que se caracterizan por la acumulación de sustancias tóxicas a causa de defectos enzimáticos, conocidos como errores innatos del metabolismo (EIM), estas enfermedades son monogénicas y de herencia autosómica recesiva en su mayoría.

En la actualidad se consideran patologías tratables, lo que permite el acceso a terapias orientadas a atender los síntomas que producen; aunque su diagnóstico debe ser oportuno para evitar secuelas irreparables (Cabello \& Giugliani, 2015). 
Dentro de las categorías en que se clasifican los EIM se encuentran las alteraciones fisiológicas de los aminoácidos, denominadas aminoacidopatías. Una de las maneras en que se pueden detectar es mediante ensayos genéticos (Wasim, et. al.; 2018).

La fenilcetonuria clásica es una clase de aminoacidopatía, una enfermedad hereditaria caracterizada por la ausencia o deficiencia de la enzima fenilalanina hidroxilasa, principalmente debido a mutaciones en el gen, lo que resulta en un aumento en la concentración de fenilalanina (PHE) en la sangre (Htun, et. al.; 2015). Por ello, el objetivo del tratamiento es reducir la concentración de PHE en sangre a menos de $360 \mu \mathrm{mol} / \mathrm{L}$ para prevenir el retraso mental o daño neurológico (López-Mejía, VergaraVázquez \& Guillén-López, 2019).

Esta dieta baja en PHE consiste en dar comida vegana-vegetariana (sin carne, pescado, leche, queso, huevos, nueces, pan o productos de soya) más una fórmula alimenticia médica sintética y libre de PHE que contenga aminoácidos esenciales y no esenciales, vitaminas, oligoelementos y minerales, es decir, todo lo que requiere un individuo durante su crecimiento; dieta que se inicia en las primeras semanas de vida tras el diagnóstico y debe cumplirse durante toda la vida (Htun, et. al.; 2015). Por tanto, en esta revisión se pretende investigar sobre en qué consiste la enfermedad como tal y el tratamiento nutricional que se le debe dar a estos pacientes desde las etapas tempranas de vida según datos relevantes recientes. Se discutirá también acerca de fórmulas infantiles novedosas que pueden ser administradas $\mathrm{y}$, brevemente, sobre el impacto que esta dieta puede tener sobre la microbiota de los niños con PKU.

\section{OBJETIVO}

Evidenciar el tratamiento nutricional brindado a pacientes pediátricos con fenilcetonuria según datos recientes sobre el tema.

\section{DISCUSIÓN}

La actividad hepática de la enzima fenilalanina hidroxilasa cataliza la conversión de fenilalanina (PHE) a tirosina (TYR), esta última siendo un aminoácido (AA) indispensable en la fenilcetonuria (PKU). Si se tiene una ingesta normal de proteína dietética en un paciente con PKU no tratada sucede que la PHE se acumula en la sangre, lo que lleva a concentraciones tóxicas del AA a nivel de cerebro pudiendo causar un deterioro cognitivo profundo (Ney, et. al.; 2016). Son neurotóxicos principalmente debido a su efecto inhibitorio sobre el transporte de la barrera de sangre-cerebro de los L-aminoácidos libres que son necesarios para la síntesis de proteínas y neurotransmisores como dopamina y serotonina. Por tanto, si no se tratan, estos niños pueden presentar microcefalia, epilepsia, olor a humedad, disminución de la pigmentación de la piel y el cabello, eccema, discapacidad intelectual grave y problemas de comportamiento, así como cambios estructurales del cerebro visibles en la resonancia magnética. A pesar de los nuevos avances y estrategias de tratamiento, la intervención dietética sigue siendo el pilar del tratamiento de PKU (Verduci, et. al.; 2016). Por esta razón, como parte de la terapia deben adherirse de por vida a una dieta que limita la ingesta de PHE proveniente de los alimentos $y$, tradicionalmente, se utilizan suplementos a base de AA para proporcionar la mayoría del nitrógeno y los micronutrientes requeridos ( $\mathrm{Ney}$, et. al.; 2016).

Anteriormente, se mencionó que la PKU se suele deber a mutaciones en el gen, las cuales pueden tener varias combinaciones que dan como 
resultado un espectro completo de fenotipos metabólicos que van desde PKU severa hasta moderada y leve. En países desarrollados, esta enfermedad se identifica al nacer a través de programas de detección de recién nacidos y se clasifican por su fenotipo clínico (Verduci, et. al.; 2016). En Costa Rica, el diagnóstico se da a través de la Prueba de Tamizaje Neonatal, o prueba de talón, que se lleva a cabo en el Hospital Nacional de Niños, idealmente el tercer día de nacido (PNT, 2014).

Una vez realizado el diagnóstico para PKU, es importante iniciar el tratamiento nutricional de forma inmediata. Dependiendo de los niveles iniciales del AA en sangre puede ser necesario eliminar toda fuente de PHE de la dieta, ofreciendo únicamente alimento o fórmula nutricional libre de PHE por un periodo de 24 a 96 horas; o en el caso de lactantes la ingestión se realizará a través de la leche materna o fórmula infantil, pero la cantidad de PHE que debe ingerir cada paciente como tal dependerá de la tolerancia individual la cual varía según la edad, estado nutricional, tipo de variante patogénica, actividad enzimática residual, entre otros (López-Mejía, et. al.; 2019). Aunque, en prematuros recién nacidos no hay necesidad de aportar una dieta parenteral libre de PHE al tener una alta tolerancia a este AA en gran parte por su estado catabólico (Weiss, Lotz-Havla, Dokoupil \& Maier; 2020).

Se dice que los lactantes no deben recibir lactancia materna exclusiva, más esto no quiere decir que se deba suspender, sino complementarse con una fórmula libre de PHE. Esto por cuanto, según López-Mejía, et. al. (2019), existe evidencia de que los pacientes con PKU alimentados con seno materno mantienen un adecuado incremento ponderal, así como niveles de PHE más favorables en comparación con aquellos que no la reciben. Adicionalmente, han surgido publicaciones que no solo sitúan a la lactancia materna como una de las herramientas de prevención de sobrepeso y obesidad en pacientes con $\mathrm{PKU}$, sino que también la asocian a un coeficiente intelectual más elevado (LópezMejía, et. al.; 2019).

Ahora, en cuanto a la introducción de alimentos sólidos se deben evitar los alimentos ricos en proteínas como carne, pescado, huevos, productos lácteos, pan, nueces y semillas. Por lo tanto, esta dieta comprende principalmente de alimentos bajos en proteínas como vegetales, frutas y algunos cereales, con el fin de alcanzar los niveles ideales de PHE necesarios para los procesos de crecimiento (Verduci, et. al.; 2016). En niños sanos, la OMS recomienda iniciar alimentación complementaria a partir de los 6 meses, sin embargo, en el escrito de LópezMejía, et. al. (2019) se menciona que introducir sólidos un poco antes de los 6 meses de vida (5 meses) puede ser benéfico, ya que existe menor respuesta neofóbica a los alimentos y puede haber una mejor aceptación de la comida. Aun así, la decisión de cuándo iniciarla es un aspecto individualizado por el motivo de que se deben tomar en cuenta aspectos como: adecuado sostén cefálico, disminución de la protrusión de la lengua, desarrollo de movimientos de mano a boca, función neurológica, entre otros.

Asimismo, la cantidad de verduras, frutas y cereales que se administren deben ser en cantidades específicas previamente calculadas para ofrecer una cantidad determinada de PHE que tolere el paciente. De esta manera, es un proceso que requiere de especial atención, ya que los padres además de aprender el proceso de introducción de alimentos habitual requieren de un entrenamiento especial para aprender a cuantificar la PHE o cantidad de proteína que 
ingieren sus hijos, por lo que la intervención de un profesional en nutrición especialista en PKU es fundamental (López-Mejía, et. al.; 2019).

López-Mejía, et. al. (2019) indican que el cálculo dietético de las cantidades de fórmula y alimentos que requiere el paciente se debe hacer en función de las necesidades de proteína por edad, tomando como base las recomendaciones específicas de proteína para esta enfermedad. De acuerdo con Verduci, et. al. (2016), la cantidad total requerida de ingesta diaria de proteínas se obtiene consumiendo sustitutos de proteínas libres de PHE adicionales que suministran aminoácidos esenciales en proporciones adecuadas. Este tipo de régimen dietético generalmente incluye un alto contenido de carbohidratos, bajo contenido de grasas saturadas (menos de $7 \mathrm{~g}$ ) y grasas poliinsaturadas de cadena larga y colesterol (menos de $50 \mathrm{mg}$ ). Características que pueden parecerse a las de una dieta vegana con respecto a la composición de los alimentos permitidos.

Ahora bien, el patrón de este tipo de dieta puede sugerir que se lleguen a representar ciertas deficiencias. Por ejemplo, como estos pacientes nunca consumen pescado, se puede provocar una deficiencia crónica de ácidos grasos omega-3 y omega- 6 con el riesgo de cambios ateroscleróticos tempranos (Htun, et. al.; 2015). Sin embargo, en el artículo de Verduci, et. al. (2016) se discute la posible relación de la dieta restringida de PHE en niños con PKU con factores de riesgo cardiovascular, para lo cual según la literatura evaluada, aunque los niños con PKU que cumplen con la dieta pueden exhibir niveles más bajos de colesterol total y colesterol LDL en sangre que la población sana, no parecen mostrar riesgos cardiovasculares diferentes; pero la literatura existente es escasa. Por otra parte, Htun, et. al. (2015) estudiaron a
43 pacientes con $\mathrm{PKU}$ y 58 sanos para evaluar el impacto que podía tener en el perfil lipídico el no consumo de ácidos grasos omega-3 y omega-6 por medio de pescados. El colesterol HDL fue significativamente menor en el grupo de PKU en comparación con los controles, pero en un rango normal. No hubo diferencia entre el colesterol LDL y total. El nivel de ácidos grasos omega- 6 y omega- 3 en las membranas de los glóbulos rojos no difirió significativamente entre los grupos. Estos resultados no se debieron a la falta de cumplimiento de la dieta ya que los niveles sanguíneos de PHE siempre estuvieron dentro de los límites recomendados. Por lo tanto, parece que los pacientes con PKU compensan con éxito la falta de ingesta de ácidos grasos omega-3 causada por una dieta libre de pescado con la ingesta de aceites vegetales especiales como, por ejemplo, aceite de canola o aceite de linaza. O bien, se podría argumentar que no hubo diferencia porque la ingesta de pescado graso también es baja en el grupo de control saludable. En general, hallaron que la dieta libre de pescado no induce a cambios ateroscleróticos tempranos y activación plaquetaria en pacientes con PKU (Htun, et. al.; 2015).

Por otro lado, de acuerdo con Ney, et. al. (2016), el cumplimiento de esta dieta es pobre y hay evidencia de resultados de salud subóptimos en pacientes que usan los suplementos a base de AA incluyendo insuficiencia neurocognitiva, fragilidad esquelética y función renal alterada. Esto concuerda con el artículo de Pinto, et. al. (2017), quien añade que su sabor amargo se asocia con el rechazo. Por esta razón, coinciden en que se necesitan nuevas opciones para proporcionar una alternativa que mejore el cumplimiento de por vida de la dieta baja en PHE. Apartirde esto, sehan realizado diversos estudios que analizan el uso del glicomacropéptido para el tratamiento de $\mathrm{PKU}$, el cual es la única proteína conocida que no contiene PHE y se puede 
convertir en una variedad de productos con sabor. Asimismo, es aislada del suero de queso y ha mostrado propiedades prebióticas, por lo que se utiliza en fórmulas infantiles, de salud gastrointestinal y obesidad (Ney, et. al.; 2016). De hecho, Bassanini, et- al. (2019) incluyen que al tener propiedades prebióticas aporta efectos beneficiosos en la microbiota intestinal.

El artículo de Ney, et. al. (2016) demostró que es una opción aceptable para el manejo nutricional de la PKU, lo que puede mejorar la adherencia de por vida a esta dieta tras el estudio con 30 niños con PKU mayores de 12 años. Añaden además, que se necesita mayor investigación para comprender cómo la ingestión de esta proteína afecta el metabolismo de treonina (THR) y la microbiota intestinal. Ahora, a pesar de que no es una proteína completa al contener cantidades limitantes de algunos AA esenciales para la PKU (específicamente arginina, histeína, leucina, triptófano y tirosina), las concentraciones de isoleucina (ILE) y THR son 2-3 veces mayores que las encontradas en otras proteínas de la dieta. Concluyen, entonces, que estas fórmulas muestran eficacia en el control de las concentraciones de PHE en sangre a lo largo del tiempo, al mismo tiempo que demuestran ventajas en cuanto al sabor, la conveniencia y la reducción de los efectos secundarios gastrointestinales (Ney, et. al.; 2016).

Esto coincide con el estudio de Pinto, et. al. (2017), quienes tras evaluar a 11 pacientes con $\mathrm{PKU}$ hallaron mejoras en las concentraciones de tirosina (TYR) en sangre y no hubo deterioro en el control de PHE en sangre a pesar de que el glicomacropéptido aportaba $34 \mathrm{mg} /$ día adicionales de este $A A$. Los resultados de la TYR no habían sido encontrados antes, por lo que especulan que la mejor absorción del glicomacropéptido puede ayudar a mejorar los niveles de TYR en sangre, el cual es esencial en esta enfermedad al correlacionarse con mejores habilidades neurocognitivas y mejores niveles de dopamina. Además, no encontraron cambios en la composición corporal o marcadores nutricionales y aunque la ingesta total de energía fue menor cuando los pacientes tomaban fórmulas con glicomacropéptido, esta diferencia no fue significativa. Por ello, se puede decir que tiene un efecto más saciante debido a su absorción más lenta. Este estudio tuvo varias limitaciones como que la cantidad de pacientes fue un número pequeño, la gravedad de PKU era variable y algunos recibían glicomacropéptido solo en parte, por lo cual, sería oportuno investigar sobre más información al respecto.

Por otro lado, Bassanini, et. al. (2019) discuten que a pesar de que los niños con PKU tienen una mayor ingesta de vegetales y fibra, su perfil microbiano intestinal es diferente de aquellos individuos que consumen una dieta alta en fibra y baja en proteínas. En su estudio con 42 niños investigaron el impacto de una dieta baja en PHE en la microbiota y sus posibles consecuencias en el bienestar de los pacientes. Encontraron que la edad en que se comienza esta dieta corresponde con una etapa crucial en la adquisición y maduración de la microbiota, donde los factores ambientales pueden tener un profundo impacto. Como resultado, se encontró que la microbiota se agotó en especies productoras de butirato (considerado beneficioso) y se enriqueció en géneros conocidos por ejercer un efecto proinflamatorio en la mucosa intestinal. Por tanto, no están obteniendo los beneficios conocidos de llevar una dieta alta en fibra y vegetales.

\section{CONCLUSIÓN}

Es necesario hacer las pruebas de tamizaje al nacimiento de los niños con el fin de obtener 
un diagnóstico de esta enfermedad lo antes posible, lo cual ayudaría a prevenir posibles daños neurológicos irreversibles. Una vez que se cumple este paso, se debe incluir a un profesional en nutrición dentro del equipo multidisciplinario para comenzar el tratamiento lo más antes posible. Adicionalmente, se encontró que los glicomacropéptidos se pueden convertir en una buena opción de fórmula nutricional que aporta beneficios tanto organolépticos, como nutricionales y prebióticos que promuevan el buen estado de salud de los niños con PKU al mismo tiempo que permita conllevar esta dieta de por vida de mejor manera.

\section{REFERENCIAS}

1. Bassanini, G., Ceccarani, C., Borgo, F., Severgnini, M., Rovelli, V., Morace, G., Verduci, E. \& Borghi, E. (2019). Phenylketonuria diet promotes shifts in Firmicutes populations. Frontiers in cellular and infection microbiology, 9, 101.

2. Cabello, J. F. \& Giugliani, R. (2015). Errores innatos del metabolism. Revista médica clínica CONDES, 26(4), 483-486.

3. Htun, P., Nee, J., Ploeckinger, U., Eder, K., Geisler, T., Gawaz, M., Bocksch, W. \& Fateh-Moghadam, S. (2015). Fish-free diet in patients with phenylketonuria is not associated with early atherosclerotic changes and enhanced platelet activation. PLoS One, 10 (8).

4. López-Mejía, L., Vergara-Vázquez, M., \& Guillén-López, S. (2019). ¿Qué aspectos considerar al iniciar el tratamiento nutricional para Fenilcetonuria? Acta Pediátrica de México, 39(S1), 6674.

5. Ney, D. M., Stroup, B. M., Clayton, M. K., Murali, S. G., Rice, G. M., Rohr, F., \& Levy, H. L. (2016). Glycomacropeptide for nutritional management of phenylketonuria: a randomized, controlled, crossover trial. The American journal of clinical nutrition, 104(2), 334-345.

6. Pinto, A., Almeida, M. F., Ramos, P. C., Rocha, S., Guimas, A., Ribeiro, R., Martins, A., MacDonald, A. \& Rocha, J. C. (2017). Nutritional status in patients with phenylketonuria using glycomacropeptide as their major protein source. European journal of clinical nutrition, 71(10), 1230-1234.

7. PNT (2014). Programa Nacional de Tamizake Neonatal, Costa Rica [página web].

Verduci, E., Banderali, G., Moretti, F., Lassandro, C., Cefalo, G., Radaelli, G., Salvatici, E. \& Giovannini, M. (2016). Diet in children with phenylketonuria and risk of cardiovascular disease: a narrative overview. Nutrition, Metabolism and Cardiovascular Diseases, 26(3), 171-177.

8. Wasim, M., Awan, F. R., Khan, H. N., Tawab, A., lqbal, M. \& Ayesha, H. (2018). Aminoacidopathies: prevalence, etiology, screening, and treatment options. Biochemical Genetics, 56, 7-21.

9. Weiss, K., Lotz-Havla, A., Dokoupil, K., \& Maier, E. M. (2020). Management of three preterm infants with phenylketonuria. Nutrition, 71, 110619. 